'Fundação Oswaldo Cruz (Fiocruz), Centro de Pesquisa Aggeu Magalhães - Recife (PE), Brasil. sydia@cpqam.fiocruz.br

2 Université de Montréal, École de santé publique de I'Université de Montréal Montreal (QC), Canadá. louise.potvin@umontreal.ca

3 Universidade Federal da Bahia (UFBA), Instituto de Saúde Coletiva - Salvador (BA), Brasil

medina@ufba.br

\section{Sustentabilidade de intervenções em promoção da saúde: uma sistematização do conhecimento produzido}

\author{
Sustainability in health promotion interventions: a systematization of \\ the produced knowledge
}

Sydia Rosana de Araujo Oliveira', Louise Potvin², Maria Guadalupe Medina

RESUMO Foi realizada revisão da literatura com intuito de sistematizar o conhecimento produzido no campo da promoção da saúde em relação ao tema sustentabilidade. As bases de dados consultadas foram Lilacs, SciELO e Web of Science, no período entre 1989 e 2014. O corpus contou com 35 artigos, sendo analisados conceito de sustentabilidade, metodologia e resultados dos estudos. Verificou-se que os estudos têm se dedicado a encontrar fatores que influenciam a sustentabilidade de intervenções de saúde, no entanto, não há evidências sobre que fatores são suficientes para a sustentabilidade. Conclui-se que há pouca literatura sobre o tema em questão no âmbito nacional e se recomenda novas investigações.

PALAVRAS-CHAVE Institucionalização; Avaliação em saúde; Programa de promoção da saúde; Revisão.

ABSTRACT Scientific review was performed aiming to systematize the knowledge produced in the field of health promotion, regarding sustainability. Databases consulted were Lilacs, SciELO and Web of Science, in the period between 1989 and 2014. The corpus included 35 papers being analyzed the concept of sustainability, methodology and studies results. It was found that the studies have been devoted to finding factors that influence the sustainability of health interventions, however, there is no scientific evidence about which factors are sufficient for the sustainability. It was concluded that there is little literature on the subject in question at the national level and further investigations are recommended.

KEYWORDS Institutionalization; Health evaluation; Health promotion program; Review. 


\section{Introdução}

A compreensão da dinâmica e implementação das intervenções de saúde tem sido objeto de interesse de diversos pesquisadores nas últimas décadas. Entre as abordagens e modelos explicativos formulados para entender o processo de elaboração e desenvolvimento das intervenções e, em especial, das políticas de saúde, podem-se destacar as teorias da escolha racional, do incrementalismo, da análise de sistemas, da coalização de defesa, da teoria da escolha pública e do ciclo das políticas públicas (KELLY; PALUMBO, 1992; SOUZA, 2006).

A partir da constituição de 1988, com o processo de reorientação das políticas públicas no governo brasileiro, os estudos relacionados ao ciclo das políticas ganharam impulso (CAPELLA, 2006; MACHADO, 2013; PINTO, 2008). As dificuldades na implementação de novas práticas suscitaram o questionamento quanto à compreensão do processo pelo qual as políticas públicas em saúde 'acontecem', isto é, o entendimento de como e por que os governos optam por determinadas ações (PINTO, 2008; SOUZA, 2006; VIANA, 1996). No entanto, uma questão fundamental ainda não foi investigada de forma satisfatória no País: $\mathrm{O}$ que determina a manutenção ou o fim de uma intervenção?

Estudos relacionados ao ciclo de vida das intervenções (BOPP; SAUNDERS; LATTIMORE, 2013; SCHEIRER, 2005) incorporam ao ciclo das políticas um novo estágio: a sustentabilidade. Embora venha despertando bastante interesse e esteja sendo amplamente discutida em diversos países - de modo especial em relação às intervenções que se referem à promoção de saúde -, a sustentabilidade não se constitui uma questão sobre a qual atores governamentais e sociais concentrem sua atenção no Brasil, mesmo quando os recursos para a promoção da saúde são limitados e as expectativas quanto ao que pode ser alcançado são elevadas.

Para melhor compreender o estado da arte em matéria de sustentabilidade, este estudo - produto da tese intitulada 'Sustentabilidade da estratégia saúde da família: o caso de um município baiano' objetiva sistematizar a contribuição dos estudos produzidos na literatura científica no campo da saúde pública/saúde coletiva em relação ao tema, especialmente no que se refere a intervenções de promoção da saúde, buscando responder às seguintes questões de investigação: Como o campo da saúde pública/saúde coletiva tem abordado o tema da sustentabilidade nas intervenções de promoção da saúde? E quais os fatores que facilitam e dificultam a sustentabilidade nas intervenções de promoção da saúde?

\section{Método}

Foi realizada revisão de literatura sobre o tema sustentabilidade em saúde. A elaboração deste estudo foi realizada seguindo as seguintes etapas: 1) identificação do tema e elaboração da questão norteadora; 2) busca ou amostragem na literatura; 3) coleta de dados; 4) análise crítica dos estudos incluídos; 5) interpretação dos resultados; e 6) apresentação da revisão/síntese do conhecimento (SOUZA; SILVA; CARVALHO, 2010).

Foram incluídos estudos divulgado em periódicos publicados em bases de dados bibliográficas informatizadas (Literatura Latino-Americana e do Caribe em Ciências da Saúde - Lilacs; Scientific Electronic Library Online - SciELO; e Web of Science), utilizando-se os descritores e operadores boleanos 'sustentabilidade OR institucionalização OR rotinização AND saúde OR Intervenção de saúde OR Programas de promoção da saúde', em português e seus correlatos em inglês e espanhol. Além disso, empregou-se a estratégia de bola de neve, buscando-se artigos citados nos textos identificados. A coleta de dados ocorreu em novembro de 2014.

Foi construída uma base de dados única, em Excel, com o total de 475 documentos 
identificados. Excluídas as duplicações, obteve-se, então, 441 documentos que foram compilados e submetidos a processo de seleção, considerando-se os seguintes critérios de inclusão: ser artigo e ter sido publicado de 1989 a 31 de junho de 2014 nas bases de dados selecionadas; ter seu resumo disponível nas bases de dados incluídas neste estudo; ser estudo empírico e/ou de revisão e/ou conceitual na área de promoção da saúde, cujo foco principal era a sustentabilidade da intervenção; ter como objetivo a sustentabilidade das intervenções, e/ ou apresentar um dos termos descritos acima, no sentido de manutenção da intervenção após um período inicial de implementação.

Seguindo os critérios de exclusão, não foram incluídos editoriais, teses, dissertações, anais, congressos e estudos que não abordam a temática dentro dos seus objetivos. Não houve exclusão em virtude da língua.

Na primeira etapa, após a busca, foram lidos os títulos e resumos da totalidade dos documentos selecionados. Os artigos que preencheram os critérios de inclusão ou que suscitaram dúvidas quanto à sua exclusão imediata foram selecionados para a etapa seguinte. Os artigos selecionados foram lidos na íntegra e examinados pelo autor principal desta pesquisa; quando dúvidas surgiam, foi realizada discussão com outro pesquisador e resolvidas por consenso.

Trinta e cinco documentos foram incluídos nesta revisão. Os artigos compilados foram classificados, catalogados e sistematizados de acordo com instrumento de coleta de dados, permitindo verificar as seguintes características de cada pesquisa: ano e país do estudo, intervenções dos estudos, descrição do conceito, termo adotado, tipo de estudo, delineamento da pesquisa, instrumento utilizado e resultados associados à sustentabilidade.

Após essa etapa, foram realizadas a leitura crítica e a discussão dos artigos selecionados, sendo os resultados apresentados a seguir.

\section{Resultados e discussão}

Foram analisados 35 artigos (quadro 1). Entre estes, 29 (82,86\%) foram encontrados na base de dados Web of Science, apenas 1 $(2,86 \%)$ foi encontrado no SciELO e 5 artigos internacionais $(14,28 \%)$ foram encontrados a partir da estratégia bola de neve. Esses dados demonstram a escassez de publicações sobre a temática no âmbito nacional.

Quadro 1. Descrição dos estudos incluídos na revisão integrativa, segundo ano de publicação, referência, país de localização do primeiro autor, tipo de publicação e aspecto da sustentabilidade das intervenções abordado na publicação

\begin{tabular}{|c|c|c|c|c|}
\hline Ano & Autoria/Título/Periódico & País & $\begin{array}{l}\text { Tipo de } \\
\text { Publicação }\end{array}$ & $\begin{array}{l}\text { Aspecto da sustentabilidade das intervenções abordado } \\
\text { na publicação }\end{array}$ \\
\hline 1989 & $\begin{array}{l}\text { STECKLER, A.; GOODMAN, R. M. How to } \\
\text { Institutionalize Health Promotion Programs. } \\
\text { American Journal of Health Promotion }\end{array}$ & EUA & Empírico & $\begin{array}{l}\text { Revela fatores que elevam ou diminuem a } \\
\text { institucionalização de programas de promoção da saúde } \\
\text { em escolas e agencias comunitárias. }\end{array}$ \\
\hline 1993 & $\begin{array}{l}\text { GOODMAN, R. M. et al. Development of level of } \\
\text { institutionalization scales for health promotion } \\
\text { programs. Health education quarterly }\end{array}$ & EUA & Empírico & $\begin{array}{l}\text { Testa um instrumento para medir o nível de } \\
\text { institucionalização de programas de promoção da } \\
\text { saúde. }\end{array}$ \\
\hline 1998 & $\begin{array}{l}\text { O'LOUGHLIN, J. et al. Correlates of the sustainability } \\
\text { of community-based heart health promotion } \\
\text { interventions. Preventive medicine }\end{array}$ & Canadá & Empírico & $\begin{array}{l}\text { Investiga fatores relacionados à percepção de } \\
\text { sustentabilidade em programas de prevenção à saúde } \\
\text { coronariana }\end{array}$ \\
\hline 1998 & $\begin{array}{l}\text { SHEDIAC-RIZKALLAH, M. C.; BONE, L. R. Planning } \\
\text { for the sustainability of community-based health } \\
\text { programs: conceptual frameworks and future } \\
\text { directions for research, practice and policy. Health } \\
\text { education research }\end{array}$ & EUA & Teórico & $\begin{array}{l}\text { Compreende o conceito de sustentabilidade e } \\
\text { indicadores operacionais que podem ser utilizados no } \\
\text { monitoramento da sustentabilidade. }\end{array}$ \\
\hline
\end{tabular}




\begin{tabular}{|c|c|c|c|c|}
\hline 2000 & $\begin{array}{l}\text { PAINE-ANDREWS, A. Promoting Sustainability of } \\
\text { Community Health Initiatives: An Empirical Case } \\
\text { Study. Health Promotion Practice }\end{array}$ & EUA & Empírico & $\begin{array}{l}\text { Analisa as estratégias utilizadas para promover a } \\
\text { sustentabilidade de iniciativas de saúde comunitária }\end{array}$ \\
\hline 2001 & $\begin{array}{l}\text { GOODSON, P. et al. Maintaining prevention in } \\
\text { practice. American Journal of Preventive Medicine }\end{array}$ & EUA & Empírico & $\begin{array}{l}\text { Examina a institucionalização da ferramenta Prevenção } \\
\text { colocada na prática (Put Prevention Into Practice - } \\
\text { PPIP) dentro de cinco clínicas de cuidados primários }\end{array}$ \\
\hline 2003 & $\begin{array}{l}\text { ALEXANDER, J. A. et al. Sustainability of } \\
\text { collaborative capacity in community health } \\
\text { partnerships. Medical care research and review : } \\
\text { MCRR }\end{array}$ & EUA & Empírico & $\begin{array}{l}\text { Desenvolve um modelo conceitual de sustentabilidade } \\
\text { em parcerias com a comunidade e identifica potenciais } \\
\text { determinantes da sustentabilidade }\end{array}$ \\
\hline 2004 & $\begin{array}{l}\text { JOHNSON, K. et al. Building capacity and } \\
\text { sustainable prevention innovations: A sustainability } \\
\text { planning model. Evaluation and Program Planning }\end{array}$ & EUA & Revisão & $\begin{array}{l}\text { Apresenta conceito de sustentabilidade e modelo de } \\
\text { planejamento da sustentabilidade }\end{array}$ \\
\hline 2004 & $\begin{array}{l}\text { MANCINI, J. A.; MAREK, L. Sustaining Community- } \\
\text { Based Programs for Families: Conceptualization and } \\
\text { Measurement. Family Relations }\end{array}$ & EUA & Teórico & $\begin{array}{l}\text { Apresenta um modelo conceitual de sustentabilidade } \\
\text { para programa baseado na comunidade }\end{array}$ \\
\hline 2004 & $\begin{array}{l}\text { PLUYE, P. et al. Program sustainability: Focus } \\
\text { on organizational routines. Health Promotion } \\
\text { International }\end{array}$ & Canadá & Empírico & Avalia a presença de rotinas na organização. \\
\hline 2004 & $\begin{array}{l}\text { PLUYE, P.; POTVIN, L.; DENIS, J. L. Making public } \\
\text { health programs last: conceptualizing sustainability. } \\
\text { Evaluation and Program Planning }\end{array}$ & Canadá & Teórico & $\begin{array}{l}\text { Compreende sustentabilidade a partir de duas questões } \\
\text { principais: as estruturas sociais e os aspectos temporais }\end{array}$ \\
\hline 2004 & $\begin{array}{l}\text { SWERISSEN, H.; CRISP, B. R. The sustainability of } \\
\text { health promotion interventions for different levels of } \\
\text { social organization. Health Promotion International }\end{array}$ & Austrália & Teórico & $\begin{array}{l}\text { Fornece orientação para o desenvolvimento de uma } \\
\text { política de sustentabilidade de promoção da saúde }\end{array}$ \\
\hline 2005 & $\begin{array}{l}\text { NILSEN, P. et al. Towards improved understanding } \\
\text { of injury prevention program sustainability. Safety } \\
\text { Science }\end{array}$ & Suécia & Empírico & $\begin{array}{l}\text { Contribui para melhor compreensão das condições em } \\
\text { que os programas de prevenção de lesões têm maior } \\
\text { probabilidade de atingir a sustentabilidade. }\end{array}$ \\
\hline 2005 & $\begin{array}{l}\text { PLUYE, P. et al. Program sustainability begins with } \\
\text { the first events. Evaluation and Program Planning }\end{array}$ & Canadá & Empírico & $\begin{array}{l}\text { Avalia a variabilidade de eventos que marcam a } \\
\text { implementação e sustentabilidade de programas de } \\
\text { modo concomitante. }\end{array}$ \\
\hline 2005 & $\begin{array}{l}\text { SCHEIRER, M. A. Is Sustainability Possible? A } \\
\text { Review and Commentary on Empirical Studies } \\
\text { of Program Sustainability. American Journal of } \\
\text { Evaluation }\end{array}$ & EUA & Revisão & $\begin{array}{l}\text { Revisa a literatura sobre sustentabilidade de projetos } \\
\text { relacionados à saúde, focando em estudos que } \\
\text { apresentam dados coletados num ponto do tempo após } \\
\text { os fundos externos iniciais terem expirado. }\end{array}$ \\
\hline 2006 & $\begin{array}{l}\text { RIDDE, V.; PLUYE, P.; QUEUILLE, L. Évaluer la } \\
\text { pérennité des programmes de santé publique : } \\
\text { un outil et son application en Haïti. Revue } \\
\text { d'Épidémiologie et de Santé Publique }\end{array}$ & Canadá & Empírico & $\begin{array}{l}\text { Avalia o grau de perenidade de uma unidade de cuidado } \\
\text { nutricional do Haiti. }\end{array}$ \\
\hline 2008 & $\begin{array}{l}\text { GRUEN, R. L. et al. Sustainability science: an } \\
\text { integrated approach for health-programme planning. } \\
\text { The Lancet }\end{array}$ & Austrália & Revisão & $\begin{array}{l}\text { Avalia as perspectivas existentes sobre sustentabilidade } \\
\text { de programa de saúde e desenvolve um modelo de } \\
\text { sustentabilidade }\end{array}$ \\
\hline 2008 & $\begin{array}{l}\text { SAVAYA, R.; SPIRO, S.; ELRAN-BARAK, R. } \\
\text { Sustainability of Social Programs: A Comparative } \\
\text { Case Study Analysis. American Journal of Evaluation }\end{array}$ & Israel & Empírico & $\begin{array}{l}\text { Identifica características que potencializam a } \\
\text { sustentabilidade de programas. }\end{array}$ \\
\hline 2009 & $\begin{array}{l}\text { HANSON, H. M.; SALMONI, A. W.; VOLPE, R. } \\
\text { Defining program sustainability: Differing views of } \\
\text { stakeholders. Canadian Journal of Public Health }\end{array}$ & Canadá & Empírico & $\begin{array}{l}\text { Documenta o significado do termo 'sustentabilidade' } \\
\text { para as partes interessadas no planejamento e na } \\
\text { implementação de um projeto de prevenção de quedas } \\
\text { de base comunitária. }\end{array}$ \\
\hline 2010 & $\begin{array}{l}\text { FELISBERTO, E. et al. Análise da sustentabilidade de } \\
\text { uma política de avaliação: o caso da atenção básica } \\
\text { no Brasil. Cadernos de Saúde Pública }\end{array}$ & Brasil & Empírico & $\begin{array}{l}\text { Avalia a sustentabilidade da política nacional de } \\
\text { monitoramento e avaliação da atenção básica }\end{array}$ \\
\hline
\end{tabular}


Quadro 1. (cont.)

2011 HANSON, H. M.; SALMONI, A. W. Stakeholders' perceptions of programme sustainability: Findings from a community-based fall prevention programme. Public Health

2011 SCHEIRER, M. A.; DEARING, J. W. An agenda for research on the sustainability of Public Health Programs. American Journal of Public Health.

2012 SAUNDERS, R. P. et al. Assessing sustainability of Lifestyle Education for Activity Program (LEAP). Health Education Research

2012 SAVAYA, R.; SPIRO, S. E. Predictors of Sustainability of Social Programs. American Journal of Evaluation

2012 VAN ACKER, R. et al. Sustainability of the wholecommunity project "10,000 Steps": a longitudinal study. BMC public health

2012 WILTSEY STIRMAN, S. et al. The sustainability of new programs and innovations: a review of the empirical literature and recommendations for future research. Implementation Science

2013 BOPP, M.; SAUNDERS, R. P.; LATTIMORE, D. The tug-of-war: Fidelity versus adaptation throughout the health promotion program life cycle. Journal of Primary Prevention

2013 CHAMBERS, D. A; GLASGOW, R. E.; STANGE, K. C. The dynamic sustainability framework: addressing the paradox of sustainment amid ongoing change. Implementation science : IS

2013 HUIJG, J. M. et al. Factors influencing the adoption, implementation, and continuation of physical activity interventions in primary health care: a Delphi study. BMC family practice

2013 KOSKAN, A. et al. Sustainability of promotora initiatives: program planners' perspectives. J Public Health Manag Pract

2013 LOVARINI, M.; CLEMSON, L.; DEAN, C. Sustainability of community-based fall prevention programs: A systematic review. Journal of Safety Research

2013 SCHEIRER, M. A. Linking sustainability research to intervention types. American Journal of Public Health

2013 SCHELL, S. F. et al. Public health program capacity for sustainability: a new framework. Implementation science: IS

2014 WHELAN, J. et al. Cochrane Update: Predicting sustainability of intervention effects in public health evidence: Identifying key elements to provide guidance. Journal of Public Health (United Kingdom)

2014 FRIEND, S. et al. The Researchers Have Left the Building: What Contributes to Sustaining SchoolBased Interventions Following the Conclusion of Formal Research Support? Journal of school health
Canadá Empírico
Apresenta as percepções de sustentabilidade dos principais envolvidos em um programa de prevenção de queda de base comunitária.
EUA Teórico Desenvolve um paradigma para a realização de pesquisas em sustentabilidade
EUA Empírico Descreve os métodos para avaliar a sustentabilidade do programa educacional de Atividade para o estilo de vida (LEAP)
Israel Empírico Testa um modelo abrangente de preditores de três manifestações da sustentabilidade: continuação, institucionalização e duração.
Bélgica Empírico
Avalia indicadores de sustentabilidade organizacional e individual em programa de atividade física
EUA Revisão Revisa os métodos que têm sido utilizados, os resultados relatados, e os fatores que foram identificados como potenciais influências sobre o uso sustentado de novas práticas, programas ou intervenções
EUA Empírico Descreve o ciclo de vida para os programas de promoção da saúde com base em pesquisas, as principais influências de cada fase, e as questões relacionadas entre fidelidade e adaptação ao longo do processo.
EUA Teórico
Reflete sobre a sustentabilidade das intervenções e o contexto onde são desenvolvidas.

Holanda Empírico

Identifica os fatores mais relevantes para a adoção, implementação e continuação de intervenções de atividade física em cuidados de saúde primários.

EUA Empírica Analisa a sustentabilidade de iniciativas de saúde lideradas por promotoras de salud

Austrália Revisão

Identifica quaisquer teorias, modelos, quadros teóricos, influenciando fatores ou intervenções para manutenção de programas de prevenção de quedas na comunidade.

EUA Teórico Sugere um modelo para analisar a sustentabilidade de acordo com cada intervenção

EUA Teórico

Apresenta um novo quadro conceptual para a capacidade de sustentabilidade do programa para programas de saúde pública.

Austrália Revisão Compreende dez elementos-chave da sustentabilidade em intervenções de saúde pública e de promoção da saúde, usando a prevenção da obesidade baseada na comunidade.

EUA Empírico
Analisa a sustentabilidade de programa relacionado com o peso em adolescentes 
Das publicações, 18 eram dos Estados Unidos (EUA); 7 do Canadá; 4 da Austrália, havendo publicações da Bélgica, Brasil, Holanda, Israel e Suécia, as quais foram publicadas entre 1989 e 2014, sendo a maioria publicada em $2013(n=7)$.

As intervenções analisadas nos artigos diziam respeito a vários problemas ou condições relacionadas à saúde, quais sejam: obesidade, fumo e álcool, desnutrição, prevenção de quedas, prevenção de cuidados dentais, doenças crônicas, gravidez na adolescência, uso de substâncias psicoativas, violência doméstica, educação em saúde nas escolas, promoção da atividade física e promoção de saúde no trabalho.

No que se refere aos termos adotados, distintas nomenclaturas foram utilizadas para designar a manutenção das intervenções, a saber: continuação, duração, perenização, rotinização, institucionalização, incorporação, sustentabilidade, não existindo concordância na literatura em relação a uma única denominação. A dificuldade em definir esse tema, ou estabelecer um consenso em torno dele, traduziu-se com ênfases, enfoques e nuances diferenciados na literatura em análise.

Savaya e Spiro (2012) compreendem continuação como a operação ou não de um projeto e duração como a diferença de tempo entre a data de implantação ou data de término do projeto, ou ainda data de coleta dos dados para os que continuam ativos. Perenização está centrada sobre o processo que permite a continuação de atividades e de efeitos relativos ao programa (RIDDE; PLUYE; QUEUILLE, 2006), estando intimamente ligada à rotinização. Pluye et al. (2004, 2005) definiram rotinização como o processo que leva ao estabelecimento de rotinas organizacionais, as quais seriam resultados de atividades rotinizadas que se tornaram habituais. Ou ainda, o processo primário de sustentabilidade nas organizações (SHEDIAC-RIZKALLAH; BONE, 1998).

A noção de rotinização tem sido apresentada como estágio da institucionalização, referindo-se à viabilidade e integração de longo prazo de um programa e/ou atividade com a organização (SAVAYA; SPIRO, 2012; STECKLER; GOODMAN, 1989). Entretanto, pode-se entender que o termo institucionalização apresenta uma conotação mais durável que rotinização, compreendendo-se que este processo se desenvolve em camadas, sendo rotinização o primeiro nível e institucionalização o processo seguinte para a manutenção de uma intervenção em uma organização (GOODMAN ET AL., 1993; SCHEIRER, 2013). Alguns autores, ainda, conceituam institucionalização na perspectiva da continuidade atrelada a recursos financeiros que apoiam a intervenção (SAVAYA; SPIRO, 2012; STECKLER; GOODMAN, 1989).

Cabe destacar, ainda, uma fase adicional da institucionalização que é a incorporação de intervenções à organização de acolhimento. Essa etapa seria necessária quando programas surgem a partir de instituições de pesquisa ou de outras agências externas, e gradualmente são responsabilizadas por outras instituições (O'LOUGHLIN ET AL., 1998).

De acordo com Paine-Andrews et al. (2000), institucionalização e sustentabilidade são termos semelhantes, que se referem à continuação de programas ao longo do tempo e à produção de efeitos positivos na saúde. Contudo, outros estudos (FELISBERTO ET AL., 2010; PLUYE ET AL., 2005; SCHEIRER; DEARING, 2011; SCHEIRER, 2013; SHEDIACRIZKALLAH; BONE, 1998) apontam para um amplo tema da sustentabilidade, o qual abarcaria as diferentes noções de institucionalização, continuação, duração, perenização e rotinização.

Sustentabilidade, no contexto das intervenções de saúde, é definida pela manutenção ao longo do tempo de uma intervenção ou de suas atividades no âmbito organizacional e/ ou comunitário para alcançar os resultados de saúde desejados (O'LOUGHLIN ET AL., 1998), após o apoio externo da agência financiadora ter se encerrado (CHAMBERS; GLASGOW; STANGE, 2013; GRUEN ET AL., 2008; VAN ACKER ET AL., 2012). Ou ainda, o conjunto de fatores que constroem a capacidade de um programa de saúde pública manter-se ao longo do tempo (ALEXANDER ET AL., 2003; SCHELL ET AL., 2013), respondendo aos interesses 
comunitários (BOPP; SAUNDERS; LATTIMORE, 2013; MANCINI; MAREK, 2004).

Shediac-Rizkallah e Bone (1998) destacam a existência de três indicadores operacionais de sustentabilidade, são eles: 1) a manutenção dos benefícios iniciais de um programa de saúde, 2) a institucionalização do programa em um ambiente ou comunidade e 3) a construção de capacidade, que constrói recursos duráveis e permite a indivíduos ou comunidades continuarem a intervenção.

Estudos apontam uma evolução do conceito não o vinculando apenas a organizações ou comunidades, mas também podendo ser relacionado às atitudes e comportamentos dos indivíduos para manutenção das intervenções e dos seus efeitos (VAN ACKER ET AL., 2012). Esse processo dinâmico seria o estágio final da evolução de uma intervenção (PLUYE ET AL., 2005).

Em relação aos métodos utilizados, observa-se a variedade de estudos nesta pesquisa, sendo que 21 incluíram um componente empírico, destacando-se investigações de iniciativas de saúde comunitária (BOPP; SAUNDERS; LATTIMORE, 2013; FRIEND ET AL., 2014; HANSON; SALMONI; VOLPE, 2009; HANSON; SALMONI, 2011; HUIJG ET AL., 2013; KOSKAN ET AL., 2013; NILSEN ET AL., 2005; O'LOUGHLIN ET AL., 1998; PAINE-ANDREWS, 2000; SAVAYA; SPIRO; ELRAN-BARAK, 2008), avaliações de políticas e parcerias comunitárias (ALEXANDER ET AL., 2003; FELISBERTO ET AL., 2010; PLUYE ET AL., 2004; SAUNDERS ET AL., 2012; VAN ACKER ET AL., 2012) e estudos de caso sobre propostas metodológicas explicativas do processo pelo qual as intervenções se sustentam (GOODMAN ET AL., 1993; GOODSON ET AL., 2001; PLUYE ET AL., 2005; RIDDE; PLUYE; QUEUILLE, 2006; SAVAYA; SPIRO, 2012; STECKLER; GOODMAN, 1989).

Entre os estudos não empíricos, seis eram artigos de revisão (GRUEN ET AL., 2008; JOHNSON ET AL., 2004; LOVARINI; CLEMSON; DEAN, 2013; SCHEIRER, 2005; WHELAN ET AL., 2014; WILTSEY STIRMAN ET AL., 2012) e oito eram ensaios teóricos (CHAMBERS; GLASGOW; STANGE, 2013; MANCINI; MAREK, 2004; PLUYE; POTVIN; DENIS, 2004; SCHEIRER; DEARING, 2011; SCHEIRER, 2013; SCHELL ET AL., 2013; SHEDIAC-RIZKALLAH; BONE, 1998; SWERISSEN; CRISP, 2004), sendo que desses, três apresentavam uma proposta de desenvolvimento de ferramentas metodológicas de mensuração da sustentabilidade (MANCINI; MAREK, 2004; SCHELL ET AL., 2013; SHEDIAC-RIZKALLAH; BONE, 1998). Entre os estudos de desenvolvimento de ferramentas para avaliar a sustentabilidade das intervenções, em nenhum deles foi testada com sucesso a confiabilidade ou validade nem as medidas reanalisadas em estudos posteriores.

A mensuração da sustentabilidade entre os estudos analisados foi empregada com formas de aferição e medidas diferenciadas. O'Loughlin et al. (1998) e Pluye et al. (2004) avaliaram a sustentabilidade dos programas na organização de acolhimento por meio de entrevistas com informantes-chave. Outros trabalhos analisaram a sustentabilidade pelo 'nível de institucionalização' ou sustentabilidade (GOODMAN ET AL., 1993; GOODSON ET AL., 2001; MANCINI; MAREK, 2004; STECKLER; GOODMAN, 1989), OU ainda pelo uso dos eventos críticos (PLUYE ET AL., 2005; FELISBERTO ET AL., 2010).

Scheirer e Dearing (2011) apontam que, para a maioria dos estudos de sustentabilidade, uma abordagem não experimental deve ser empregada, pois ela examina as relações e os processos que relacionam as variáveis independentes relevantes para um ou mais resultados de sustentabilidade. Esses autores defendem que as pesquisas sobre sustentabilidade devem responder às seguintes questões: Quais as estratégias que funcionam melhor em relação a que tipos de resultados de sustentabilidade e para quais tipos de intervenções?

Quanto aos resultados encontrados, diversos estudos se propõem a analisar fatores que podem influenciar positivamente ou negativamente a sustentabilidade das intervenções. Dos estudos analisados, observou-se que dez fatores influenciavam positivamente, quais sejam:

Adaptação - A literatura sugere que intervenções que se modificam ao longo do tempo e que são flexiveis com o propósito de assegurar a eficácia têm se mostrado mais sustentáveis (GRUEN ET AL., 2008; HANSON; 
SALMONI; VOLPE, 2009; JOHNSON ET AL., 2004; SCHEIRER, 2005; SCHELL ET AL., 2013; WILTSEY STIRMAN ET AL., 2012). A capacidade de se adaptar ao contexto local e atender às necessidades da comunidade, ajustando-se para resolver questões em pauta, são uma condição crítica para a sustentabilidade (CHAMBERS; GLASGOW; STANGE, 2013; MANCINI; MAREK, 2004; PLUYE ET AL., 2004, 2005; SAUNDERS ET AL., 2012; SHEDIAC-RIZKALLAH; BONE, 1998; VAN ACKER ET AL., 2012). Assim, a intervenção deve ser desenhada com variedade de formatos, ao invés de um conjunto padronizado de atividades, no intuito de torná-la mais adaptável às especificidades de cada contexto (O'LOUGHLIN ET AL., 1998), sendo a adaptação intimamente ligada ao planejamento das intervenções. Contudo, cabe frisar que a intervenção deve produzir os efeitos iniciais desejados mesmo com o processo de adaptação (BOPP; SAUNDERS; LATTIMORE, 2013);

Aprendizagem - Intervenções com componentes de formação (profissional e não profissional) são mais propensas a ser sustentáveis do que aquelas sem esta atividade. Os sujeitos capacitados poderão continuar a proporcionar benefícios, treinando membros da comunidade e formando uma circunscrição no apoio do programa (HANSON; SALMONI, 2011; JOHNSON ET AL., 2004; PLUYE ET AL., 2005; SHEDIAC-RIZKALLAH; BONE, 1998), além de possibilitar a transferência das inovações para outros grupos, para promover a institucionalização (STECKLER; GOODMAN, 1989). Outro ponto que merece destaque é a aprendizagem organizacional, que deve visar à adaptação adequada de intervenções baseadas em avaliações e monitoramento em curso (CHAMBERS; GLASGOW; STANGE, 2013; HANSON; SALMONI; VOLPE, 2009; SCHEIRER, 2013; SCHELL ET AL., 2013; WHELAN ET AL., 2014; WILTSEY STIRMAN ET AL., 2012);

Avaliação - avaliar a intervenção é essencial e começa durante o planejamento. As conclusões da avaliação devem informar a evolução das intervenções; gerar práticas baseadas em evidências; e ser divulgadas e comunicadas aos públicos relevantes (CHAMBERS; GLASGOW; STANGE, 2013; SCHEIRER, 2005; SCHELL ET AL., 2013; WHELAN ET AL., 2014; WILTSEY STIRMAN ET AL., 2012). O monitoramento das intervenções pode assegurar financiamentos futuros e permitir o engajamento de profissionais-chave na intervenção (MANCINI; MAREK, 2004);

Compromisso e apoio das organizações - As organizações que acolhem a intervenção devem apoiá-la, especificamente no que tange a 'ter um ambiente político interno favorável' e existência de apoio não financeiro (JOHNSON ET AL., 2004; PLUYE ET AL., 2004; SAVAYA; SPIRO, 2012; SAVAYA; SPIRO; ELRAN-BARAK, 2008; SCHELL ET AL., 2013; STECKLER; GOODMAN, 1989). A literatura sugere a adesão da intervenção a uma política principal e/ou estruturas formais no intuito de fortalecê-la (GOODSON ET AL., 2001; JOHNSON ET AL., 2004; SWERRISEN; CRISP, 2004; FELISBERTO ET AL., 2010; SAUNDERS ET AL., 2011; FRIEND, S. ET AL., 2014; WHELAN ET AL., 2014) e a integração do programa com as atividades ou missão da organização (STECKLER; GOODMAN, 1989; O'LOUGHLIN ET AL., 1998; PAINE- ANDREWS ET AL. 2000; PLUYE ET AL., 2005; SCHEIRER, 2005; BOPP; SAUNDERS; LATTIMORE, 2013), pois programas verticais têm menos possibilidade de sustentabilidade que programas integrados (SHEDIAC-RIZKALLAH; BONE, 1998);

Defensores/Mediadores - Pesquisas consistentemente apontam a importância de líderes e a existência de defensores (pessoas influentes e proativas dentro ou fora da intervenção) no processo de sustentabilidade. Diversos autores sugerem a necessidade de cultivar atores estratégicos para que estes possam lutar pelas intervenções em momentos de maior vulnerabilidade delas (STECKLER; GOODMAN, 1989; O'LOUGHLIN ET AL., 1998; SHEDIAC-RIZKALLAH; BONE, 1998; GOODSON ET AL., 2001; JOHNSON ET AL., 2004; PLUYE; POTVIN; DENIS, 2004; MANCINI; MAREK, 2004; SCHEIRER, 2005; VAN ACKER ET AL., 2012; CHAMBERS ET AL., 2013). Savaya et al. (2008, 
2012) indicam que a chave para a sustentabilidade é a liderança na organização que acolhe a intervenção;

Equipe da intervenção - O'loughlin et al. (1998) e Paine-Andrews et al. (2000) sugerem que a formação da força de trabalho e as características da equipe têm estreita relação com a sustentabilidade da intervenção. Destaca-se o envolvimento da equipe na tomada de decisão e nas negociações concernentes à intervenção, assim como a integração de pessoal (MANCINI; MAREK, 2004; BOPP; SAUNDERS; LATTIMORE, 2013; FRIEND, S. ET AL. 2014). A literatura sobre sustentabilidade aponta, ainda, que a estabilidade da força de trabalho é determinante para a sustentabilidade (SCHEIRER, 2005; JOHNSON ET AL., 2004; WILTSEY-STIRMAN ET AL., 2012);

Financiamento - Muitas intervenções de promoção da saúde não conseguem se tornar sustentáveis devido à insuficiência de recursos (STECKLER; GOODMAN, 1989; SHEDIACRIZKALLAH; BONE, 1998; JOHNSON ET AL., 2004; NILSEN ET AL., 2005; VAN ACKER ET AL., 2012; CHAMBERS ET AL., 2013; HUIJG ET AL., 2013; KOSKAN, A. ET AL., 2013; SCHELL ET AL., 2013; FRIEND, S. ET AL., 2014). Assim, estratégias de captação de recursos devem ser exploradas (PAINE- ANDREWS ET AL., 2000; SWERRISEN; CRISP, 2004), financiar programas já existentes (STECKLER; GOODMAN, 1989), bem como o estabelecimento de diversidade de fontes de financiamento (SAVAYA; SPIRO, 2012). Esses itens são apontados como fundamentais para sustentabilidade das intervenções. Gruen et al. (2008) ressaltam a importância de otimizar recursos, principalmente em locais com recursos limitados;

Envolvimento das partes interessadas/parcerias - O engajamento contínuo das partes interessadas aumenta o ajuste entre a intervenção e o contexto local, com consequente adaptação na evolução dos problemas que podem interferir com a sustentabilidade (HANSON; SALMONI; VOLPE, 2009; CHAMBERS ET AL.,
2013). Parcerias estratégicas têm sido citadas com frequência na literatura como fator crucial para a sustentabilidade de intervenções (PAINE-ANDREWS ET AL., 2000; ALEXANDER ET AL., 2003; SWERRISEN; CRISP, 2004; SCHEIRER, 2005; FELISBERTO ET AL., 2010; HANSON; SALMONI, 2011; SAVAYA; SPIRO, 2012; VAN ACKER ET AL., 2012; BOPP; SAUNDERS; LATTIMORE, 2013; SCHELL ET AL., 2013). Atores, que podem ser individuais, membros-chave da comunidade e organizações, devem ser aproximados da intervenção no intuito de criar redes, realizar uma 'colaboração eficaz', reforçando metas de programas e promovendo esforços coordenados (STECKLER; GOODMAN, 1989; SHEDIAC-RIZKALLAH; BONE, 1998; PLUYE; POTVIN; DENIS, 2004; SCHEIRER, 2013). O estudo de PaineAndrews (2000) demonstrou que a existência de aliança entre instituições com missão semelhante pode contribuir para apoio contínuo da equipe à intervenção;

Percepção de benefícios - Quanto mais uma intervenção for direcionada a uma necessidade real ou percebida pelos membros da equipe e/ou usuários, mais provavelmente ela será sustentada (PAINE-ANDREWS ET AL., 2000; JOHNSON ET AL., 2004; MANCINI; MAREK, 2004 SCHEIRER, 2005; HUIJG ET AL., 2013). Embora considere esse fator relevante, Chambers et al. (2013) chamam a atenção para o fato de que os estudos raramente avaliam resultados utilizando como fonte a população a quem foi dirigida a intervenção;

Planejamento - O planejamento antecipado tem sido apontado como fator que influencia a sustentabilidade (PLUYE ET AL., 2005; CHAMBERS ET AL., 2013; KOSKAN, A. ET AL., 2013; SCHELL, 2013). Whelan et al. (2014) afirmam que a sustentabilidade da intervenção deve ser planejada em todas os momentos da intervenção, destacando-se na fase de concepção da intervenção, bem como no momento de término de concessão de financiamento. Goodson et al. (2001) afirmam que neste momento de finalização do subsídio por agências externas, é necessário reavaliar 
a intervenção e repensar os novos rumos dela. Bopp, Saunders e Lattimore (2013) apontam que se deve planejar a sustentabilidade com as partes interessadas, assegurando à intervenção compatibilidade com o ambiente de acolhimento.

Entre as influências negativas ou barreiras para a sustentabilidade, observam-se: a rotatividade da equipe, a mudança das práticas médicas, o baixo nível de implementação inicial do projeto e o apoio organizacional, além da falta de treinamento para os participantes da intervenção (O'LOUGHLIN ET AL., 1998; GOODSON ET AL., 2001; SCHEIRER, 2005; FELISBERTO ET AL., 2010; KOSKAN ET AL., 2013).

Com relação ao financiamento, há uma dupla questão a ser analisada: se por um lado alguns autores ressaltam que a falta de financiamento ou recursos suficientes comprometem a sustentabilidade da intervenção (HANSON; SALMONI, 2011; KOSKAN ET AL., 2013), por outro, alguns defendem que se deve evitar a entrega de recursos financeiros a agências externas e organizações para implementação de programas (STECKLER; GOODMAN, 1989). Destacando-se que ao mesmo tempo que recursos financeiros são importantes para a sustentabilidade, estes podem pesar negativamente: quanto maior for a parcela do custo coberto pelo financiador inicial, menor a probabilidade de que o projeto seja continuado e/ou institucionalizado (SAVAYA; SPIRO, 2012; SAVAYA; SPIRO; ELRAN-BARAK, 2008).

Observa-se que a rotinização das atividades é prejudicada quando estas concorrem com outras que dão à organização uma vantagem competitiva. Da mesma forma, o reconhecimento do fracasso ou da ineficiência das atividades atua contra a sustentabilidade das intervenções (PLUYE ET AL., 2005). Ao mesmo tempo que, ao analisar parcerias comunitárias, identifica-se como fragilidade para a sustentabilidade a tênue ligação dos parceiros, isto é, entre os membros e as organizações comunitárias (AleXANDer et Al., 2003). Sendo assim, faz-se necessário envidar esforços na construção de confiança e colaboração mutua. Contudo, deve-se ter cautela para não comprometer a sustentabilidade de intervenções, tornando-se dependente de alguns indivíduos-chave (NILSEN ET AL.,2005).

Em síntese, pode-se dizer que os estudos demonstraram que a sustentabilidade é multidimensional (GRUEN ET AL., 2008), isto é, que diversos fatores estão inter-relacionados na sua produção, sem a existência de um fator principal. Compreende-se, ainda, que a presença de um ou mais fatores potencializadores, bem como das barreiras apresentadas, não determinam a sustentabilidade de intervenções.

Embora os autores tenham encontrado, nas suas investigações, evidências de fatores que contribuíram para a sustentabilidade das intervenções, a incerteza permanece sobre a forma como elas podem ser sustentadas com sucesso ao longo do tempo, dada a diversidade de definições, marcos conceituais, métodos de estudo, prazos e resultados em cada um dos estudos revisados.

\section{Conclusões}

Esta é a primeira revisão da literatura publicada no Brasil sobre a sustentabilidade das intervenções de saúde. Ela centrou-se em estudos produzidos na literatura nacional e internacional no campo da saúde pública/saúde coletiva, especialmente às intervenções de promoção da saúde, e concluiu-se que o tema tem sido muito pouco explorado no Brasil.

Identificou-se 35 estudos os quais demonstraram que sustentabilidade tem permanecido um conceito polissêmico, escapando a uma definição precisa. Evidenciase, contudo, que esta revisão permitiu maior clareza na definição do escopo de como a sustentabilidade está definida, conceituada e compreendida na literatura científica.

Sendo assim, essa nova etapa deve ser incorporada na análise de política/intervenções públicas, devendo ser pensada e discutida por 
atores governamentais no momento de sua formulação. Em meio à escassez de recursos e da crescente agenda governamental, cabe ao gestor público optar por intervenções que, além de possibilitarem uma solução oportuna para os problemas a serem enfrentados, sejam mantidas ao longo do tempo.

Observou-se um conjunto de fatores que tem potencializado e um conjunto que tem dificultado a sustentabilidade de intervenções de promoção da saúde. Contudo, não foi possível determinar, a partir dos estudos incluídos nesta revisão, quais fatores ou combinação de fatores são os mais importantes para garantir a sustentabilidade ou para levar à sua ruptura.
Assim, a incerteza permanece sobre a forma como as intervenções de saúde podem ser sustentadas com sucesso ao longo do tempo, dada a diversidade de definições, marcos conceituais, métodos de estudo, prazos e resultados em cada um dos estudos revisados.

Aponta-se a necessidade de incluir estudos empíricos realizados em programas de países de baixa renda e renda média, demonstrando a lacuna desse tipo de estudo em relação ao tema sustentabilidade. Destarte, a existência dessa lacuna no campo científico deve ser suprimida com estudos de caráter empírico que possam demonstrar diferenças ou similitudes com os diversos estudos realizados.

\section{Referências}

ALEXANDER, J. A. et al. Sustainability of collaborative capacity in community health partnerships. Medical care research and review, Thousand Oaks, v. 60, n. 4 suppl, p. 130S-160S, 2003.

BOPP, M.; SAUNDERS, R. P.; LATTIMORE, D. The tug-of-war: fidelity versus adaptation throughout the health promotion program life cycle. Journal of Primary Prevention, New York, v. 34, n. 3, p. 193-207. 2013.

CAPELLA, A. C. N. Perspectivas teóricas sobre o processo de formulação de políticas públicas. Revista Brasileira de Informação Bibliográfica em Ciências Sociais, São Paulo, v. 61, n. 1, p. 25-52, 2006.

CHAMBERS, D. A.; GLASGOW, R. E.; STANGE, K. C. The dynamic sustainability framework: addressing the paradox of sustainment amid ongoing change. Implementation science, Londres, v. 8, n. 1, p. 117, 2013.

FELISBERTO, E. et al. Análise da sustentabilidade de uma política de avaliação: o caso da atenção básica no Brasil. Cadernos de Saúde Pública, Rio de Janeiro, v. 26, n. 6, p. 1079-1095, 2010.

FRIEND, S. et al. The researchers have left the building : what contributes to sustaining school-based interventions following the conclusion of formal research support? Journal of School Health, Columbus, v. 84, n. 5, p. 326-333, 2014.
GOODMAN, R. M. et al. Development of level of institutionalization scales for health promotion programs. Health education quarterly, Thousand Oaks, v. 20, n. 2, p. 161-178. 1993.

GOODSON, P. et al. Maintaining prevention in practice. American Journal of Preventive Medicine, New York, v. 20, n. 3, p. 184-189, 2001.

GRUEN, R. L. et al. Sustainability science: an integrated approach for health-programme planning. The Lancet, Londres, v. 372, n. 9649, p. 1579-1589. 2008.

HANSON, H. M.; SALMONI, A. W. Stakeholders' perceptions of programme sustainability: Findings from a community-based fall prevention programme. Public Health, Amsterdam, v. 125 , n. 8 , p. 525-532, 2011.

HANSON, H. M.; SALMONI, A. W.; VOLPE, R. Defining program sustainability: differing views of stakeholders. Canadian Journal of Public Health, Ottawa, v. 100, n. 4, p. 304-309, 2009.

HUIJG, J. M. et al. Factors influencing the adoption, implementation, and continuation of physical activity interventions in primary health care: a Delphi study. BMC family practice, [online], v. 14, n. 1, p. 142, 2013. Disponível em: <http://www.biomedcentral.com/14712296/14/142>. Acesso em: 25 nov. 2015. 
JOHNSON, K. et al. Building capacity and sustainable prevention innovations: A sustainability planning model. Evaluation and Program Planning, [online], v. 27, n. 2, p. 135-149. 2004. Disponível em: <http://www.sciencedirect.com/science/article/pii/ S0149718904000035>. Acesso em: 25 nov. 2015.

KELLY, R. M.; PALUMBO, D. Theories of policy making. In: HAWKESWORTH, M. E.; KOCH, M. (Ed.). Encyclopedia of Government and Politics v.II. 2. ed. London, New York: Routledge, 1992. p. 643-655.

KOSKAN, A. et al. Sustainability of promotora initiatives: program planners' perspectives. Journal of Public Health Management Practice, Hagerstown, v. 19, n. 5, p. E1-9, 2013.

LOVARINI, M.; CLEMSON, L.; DEAN, C. Sustainability of community-based fall prevention programs: a systematic review. Journal of Safety Research, Elmsford, v. 47, p. 9-17, 2013.

MACHADO, S. H. S. O uso da teoria de stakeholders em uma análise da etapa de formulação da Política Nacional de Medicamentos. Revista de Administração Pública, Rio de Janeiro, v. 47, n. 3, p. 543-565, 2013.

MANCINI, J. A.; MAREK, L. Sustaining community-based programs for families: conceptualization and measurement. Family Relations, [online], v. 53, n. 4, p. 339347. 2004. Disponível em: <http://www.facsmail.org/ hdfs/fcrlweb/docs/Mancini/Mancini\%20et.\%20al\%20 2004\%20(sustaining).pdf >. Acesso em: 25 nov. 2015.

NILSEN, P. et al. Towards improved understanding of injury prevention program sustainability. Safety Science, [internet], v. 43, n. 10, p. 815-833. 2005. Disponível em: $<$ http://www.sciencedirect.com/science/article/pii/ S0925753505000809>. Acesso em: 25 nov. 2015.

O'LOUGHLIN, J. et al. Correlates of the sustainability of community-based heart health promotion interventions. Preventive medicine, v. 27, n. 5, pt. 1, p. 702-712, 1998.

PAINE-ANDREWS, A. Promoting Sustainability of Community Health Initiatives: An Empirical Case Study. Health Promotion Practice, v. 1, n. 3, p. 248-258, 2000.
PINTO, I. C. M. Mudanças nas políticas públicas: a perspectiva do ciclo de política. Revista de Políticas Públicas, São Luiz, v. 12, p. 27-36. 2008.

PLUYE, P. et al. Program sustainability: focus on organizational routines. Health Promotion International, Oxford, v. 19, n. 4, p. 489-500, 2004.

PLUYE, P. et al. Program sustainability begins with the first events. Evaluation and Program Planning, [online], v. 28, n. 2, p. 123-137. 2005. Disponível em: <http://www.sciencedirect.com/science/article/pii/ S0149718904000771>. Acesso em: 24 nov. 2015.

PLUYE, P.; POTVIN, L.; DENIS, J. L. Making public health programs last: conceptualizing sustainability. Evaluation and Program Planning, [internet], v. 27, n. 2, p. 121-133, 2004. Disponível em: <http://www.sciencedirect.com/science/article/pii/S0149718904000023>. Acesso em: 25 nov. 2015.

RIDDE, V.; PLUYE, P.; QUEUILLE, L. Évaluer la pérennité des programmes de santé publique: un outil et son application en Haïti. Revue d'Épidémiologie et de Santé Publique, [internet], v. 54, n. 5, p. 421-431, 2006. Disponível em: <http://www.sciencedirect.com/science/article/pii/ S0398762006767402>. Acesso em: 24 nov. 2015.

SAUNDERS, R. P. et al. Assessing sustainability of Lifestyle Education for Activity Program (LEAP). Health Education Research, [online], v. 27, n. 2, p. 319330. 2012. Disponível em: <http://her.oxfordjournals. org/content/early/2011/12/08/her.cyr111>. Acesso em: 25 nov. 2015 .

SAVAYA, R.; SPIRO, S. E. Predictors of Sustainability of Social Programs. American Journal of Evaluation, New York, v. 33, n. 1, p. 26-43, 2012.

SAVAYA, R.; SPIRO, S.; ELRAN-BARAK, R.

Sustainability of Social Programs: A Comparative Case Study Analysis. American Journal of Evaluation, New York, v. 29, n. 4, p. 478-493, 2008.

SCHEIRER, M. A. Is sustainability possible? A review and commentary on empirical studies of program 
sustainability. American Journal of Evaluation, New

York, v. 26, n. 3, p. 320-347. 2005.

SCHEIRER, M. A. Linking sustainability research to intervention types. American Journal of Public Health, Los Angeles, v. 103, n. 4. 2013.

SCHEIRER, M. A.; DEARING, J. W. An agenda for research on the sustainability of Public Health Programs. American Journal of Public Health, Los Angeles, v. 101, n. 11, p. 2059-2067, 2011.

SCHELL, S. F. et al. Public health program capacity for sustainability: a new framework. Implementation science, Londres, v. 8, n. 1, p. 15. 2013.

SHEDIAC-RIZKALLAH, M. C.; BONE, L. R. Planning for the sustainability of community-based health programs: conceptual frameworks and future directions for research, practice and policy. Health education research, Oxford, v. 13, n. 1, p. 87-108. 1998.

SOUZA, C. Políticas públicas: uma revisão da literatura. Sociologias, Porto Alegre, n. 16, p. 20-45, 2006.

SOUZA, M. T.; SILVA, M. D.; CARVALHO, R. Revisão Integrativa: o que é e como fazer. Einstein, São Paulo, v. 8, n. 1, pt. 1, p. 102-106, 2010.

STECKLER, A.; GOODMAN, R. M. How

to institutionalize health promotion programs. American Journal of Health Promotion, Los Angeles, v. 3, n. 4, p. 34-43, 1989.

SWERISSEN, H.; CRISP, B. R. The sustainability of health promotion interventions for different levels of social organization. Health Promotion International, Oxford, v. 19, n. 1, p. 123-130, 2004.

VAN ACKER, R. et al. Sustainability of the whole-community project "10,000 Steps": a longitudinal study. BMC public health, [internet], v. 12, n. 1, p. 155, jan. 2012. Disponível em: <http://www.biomedcentral.com/14712458/12/155>. Acesso em: 25 nov. 2015.

VIANA, A. L. Abordagens metodológicas em políticas públicas. Revista de Administração Pública, Rio de Janeiro, v. 30, n. 2, p. 5-43. 1996.

WHELAN, J. et al. Cochrane Update: Predicting sustainability of intervention effects in public health evidence: Identifying key elements to provide guidance. Journal of Public Health (Oxford, England), Oxford, v. 36, n. 2, p. 347-351, 2014.

WILTSEY STIRMAN, S. et al. The sustainability of new programs and innovations: a review of the empirical literature and recommendations for future research. Implementation Science, Londres, v. 7, n. 1, p. 17, 2012.

Recebido para publicação em maio de 2015

Versão final em setembro de 2015

Conflito de interesses: inexistente

Suporte financeiro: não houve 